\title{
Improving the documentation of female genital mutilation or cutting (FGM/C) abandonment interventions and their evaluations
}

Caroline W. Kabiru

Population Council

Follow this and additional works at: https://knowledgecommons.popcouncil.org/departments_sbsr-rh

Part of the Demography, Population, and Ecology Commons, Family, Life Course, and Society Commons, Gender and Sexuality Commons, International Public Health Commons, and the Medicine and Health Commons How does access to this work benefit you? Let us know!

\section{Recommended Citation}

Kabiru, Caroline W. 2020. "Improving the documentation of female genital mutilation or cutting (FGM/C) abandonment interventions and their evaluations," Evidence to End FGM/C: Research to Help Girls and Women Thrive. New York: Population Council. 
The Evidence to End FGM/C programme consortium generates evidence to inform and influence investments, policies, and programmes for ending female genital mutilation/ cutting in different contexts.

Population Council Lead Institution

Africa Coordinating Centre for the Abandonment of Female Genital

Mutilation/Cutting, Kenya (ACCAF)

Global Research and Advocacy Group, Senegal (GRAG)

MannionDaniels Ltd. (MD)

Population Reference

Bureau (PRB)

University of Washington (Prof. Bettina Shell-Duncan)

University of California, San Diego (Dr. Gerry Mackie)

\section{Funded by}

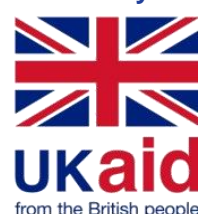

\section{Improving the documentation of female genital mutilation or cutting (FGM/C) abandonment interventions and their evaluations}

Female genital mutilation or cutting (FGM/C) is internationally recognised as a violation of human rights. ${ }^{1}$ Consequently, there have been extensive efforts to promote the abandonment of the practice. There is limited evidence on the impact of many of the efforts in part because many interventions are implemented by small organisations with inadequate resources to document and evaluate their activities. ${ }^{2}$ The complex nature of FGM/C interventions, as with other interventions aimed at addressing violence against women and girls, ${ }^{3}$ also makes it difficult to adequately document what is done, how, when, and with what results. Nonetheless, it is important that implementing organisations make every effort to document and evaluate their interventions, and share the outcomes and lessons learnt to ensure accountability and for others to learn from, adapt, replicate, and scale up successful interventions.

In this note, we provide guidance to help organisations that are implementing FGM/C abandonment interventions better document and report on their programmatic and evaluation activities. We highlight specific aspects that should be documented, drawing our guidance primarily from the World Health Organization's Programme Reporting Standards for Sexual, Reproductive, Maternal, Newborn, Child and Adolescent Health. ${ }^{4}$ The guidance is also informed by the Recommendations from the Workgroup for Intervention Development and Evaluation Research (WIDER) on reporting of behaviour-change interventions, ${ }^{5}$ and the Pan American Health Organization's Guide to Document Health Promotion Initiatives. ${ }^{6}$

We provide an outline of the key aspects that should be documented. Ideally, the documentation process should begin in the design phase and continue throughout the life of the intervention to avoid recall bias and inaccuracies. As information may appear in various documents including proposals, progress and evaluation reports, financial records, logical frameworks or theories of change, and activity manuals, all available documents should be reviewed. Ideally, implementing organisations should consider reporting all the aspects highlighted below in a concise report that can be easily shared. 


\section{Outline of documentation for FGM/C interventions and their evaluation}

\section{INTRODUCTION}

The rationale, goals and objectives, and geographic coverage (or context) of the intervention should be described to ensure that others can understand why the intervention was designed, why it was implemented in a particular setting, and what outcomes were expected.

In describing the context, it is useful to provide information on aspects such as the extent of the problem, as well as sociocultural, socioeconomic, and political attributes that are relevant to the programme. These aspects could include the prevalence of $F G M / C$ in the specific context; when, where, and how the community(ies) practise FGM/C (for example, is FGM/C practised as a rite of passage to adulthood or are girls cut when they are infants?); whether there are laws against FGM/C; and whether there are other existing FGM/C abandonment activities.

\section{OVERVIEW OF THE INTERVENTION}

Provide a summary of the intervention including the following:

- The theory of change or theoretical model that clarifies the pathways through which the intervention is expected to produce change. Defining a theory of change is critical because it helps implementers and/or evaluators select appropriate indicators of change. ${ }^{2}$

- Characteristics of the target population including geography, age, gender, marital status, and other sociodemographic characteristics to describe those expected to benefit from the intervention.

- Description of the integration of gender (e.g., does the intervention target boys, girls, men, and women?), equity (e.g., are people with disabilities included?), human rights, and ethical considerations (e.g., what measures have been put in place to protect those who participate in programmes).

- The intervention's start and end dates, noting any shifts and delays from planned timelines and the reasons for these shifts.
- The names of the implementing organisation(s), and any partners (and their roles) to describe those supporting the implementation of the intervention.

- The name of the funding institution.

- Budget and other resources, including nonfinancial contributions, such as venues, volunteers, and other in-kind contributions.

\section{DETAILED INTERVENTION ACTIVITIES}

The detailed description of intervention activities should enable others to understand how activities were designed and implemented. The level of detail should be adequate to guide replication or scale up. Key elements of the documentation of intervention activities include descriptions of the following:

- Rationale for selecting specific activities including any inputs from stakeholders and target beneficiaries.

- The setting for the intervention (e.g., schools, community, churches, mosques).

- Any pilot activities (how, when, where, by whom, and with what results).

- The core intervention components or activities (note: for interventions with multiple activities, details should be provided for each activity).

- Types of activities (e.g., training of trainers on alternative rites of passage, advocacy campaigns, training of health workers on $\mathrm{FGM} / \mathrm{C}$ ).

- Mode of delivery (e.g., face-to-face workshops, webinars, media campaigns).

- Frequency or duration of activity (e.g., two-hour sessions once a week with girls over a one-year period).

- Implementing personnel including qualifications, relevant sociodemographic characteristics, and responsibilities.

- Resources used (e.g., training curriculum), how they were developed, and how they can be accessed.

- Approaches used for implementation of quality assurance (e.g., supervisory visits). 


\section{MONITORING AND EVALUATION}

As outlined in DfID's Guidance on Monitoring and Evaluation for Programming on Violence against Women and Girls, ${ }^{3}$ monitoring and evaluating FGM/C interventions is important for assessing their impact and value for money. Monitoring and evaluating interventions also make it possible to learn from others' experiences in intervention design and implementation and ensure accountability to funders, beneficiaries, and other stakeholders.

Key elements of the documentation of monitoring activities include:

- Approaches for monitoring activity implementation including qualitative and/or quantitative data-collection methods and analyses of monitoring indicators.

- Description of the acceptability and feasibility of the intervention and how these were assessed.

- Description of the extent to which the intervention was delivered vis-à-vis the plan (i.e., fidelity of the implementation) and any adaptations made.

- Any factors that hindered or facilitated the implementation of the intervention (e.g., level of support for FGM/C abandonment by local leaders).

- Summary of all costs (financial, physical and human) required to implement activities.

- The coverage or reach of each activity disaggregated by relevant sociodemographic characteristics (e.g., number of girls participating in alternative rites of passage workshop, number of women and men attending public declaration events).

- Information on targeted individuals who fail to participate or drop out and the reasons for this attrition and any measures taken to reach out to those who have dropped out or who are not reached.

Key elements of the documentation of evaluation activities include:

- Description of the evaluation methods including the research design (e.g., randomised trial, before and after study, quasi-experimental design) and the type of evaluation conducted (e.g., process and/or impact evaluation). The description of the evaluation should also include the timing, the evaluator (internal or external) and their role. Some of the key methodological issues to be considered in undertaking evaluations of $\mathrm{FGM} / \mathrm{C}$ interventions are outlined in an article by Askew. ${ }^{2}$

- Description of results (including process, output, outcome and impact indicators) disaggregated by key sociodemographic characteristics or geographical area, as well as descriptions of any unexpected results.

- The results of any cost analyses or costeffectiveness analyses.

\section{SYNTHESIS OR LESSONS LEARNT}

To facilitate learning, it is useful for intervention implementers to reflect on and document the key implications of their efforts and the lessons learnt. Key elements of this synthesis include:

- A description of the strength and weaknesses of the intervention, including reflections on what worked well and what did not.

- Reflections on the intervention's sustainability and potential for scale up and any plans for scale up.

- Considerations for adaption in different settings.

\section{Dissemination and programme uptake}

Programme implementers should consider disseminating the descriptions of interventions and key outcomes through communication mechanisms that are easily accessible to other implementers, researchers and other stakeholders. In line with WIDER recommendations, ${ }^{5}$ they should also consider sharing intervention manuals or protocols so that others can better understand the interventions. 


\section{Resources}

\section{Developing a theory of change}

Centre for Social Action Innovation Fund (CSAIF). "Guidance for developing a theory of change for your programme."

https://media.nesta.org.uk/documents/theory of change guidance for applicants .pdf

Innovations for Poverty Action. 2016. "Guiding your program to build a theory of change."

https://www.poverty-

action.org/sites/default/files/publications/Goldilocks-Deep-

Dive-Guiding-Your-Program-to-Build-Theory-of-

Change.pdf

The Catholic Agency for Overseas Development (CAFOD). "Developing a theory of change: Workshop guidance notes."

http://cafod.azurewebsites.net/Theory of Change Guida $\underline{\text { nce.pdf }}$

\section{Documentation checklist}

Programme reporting standards for sexual, reproductive, maternal, newborn, child and adolescent health. 2017.

PRS Version 1.0.

https://www.who.int/maternal child adolescent/document s/programme-reporting-standards-checklist.pdf?ua=1 (Note: organisations can complete the PRS checklist online via http://prs.srhr.org/).

\section{Monitoring and evaluating FGM/C abandonment programmes}

Askew, I. 2005. "Methodological issues in measuring the impact of interventions against female genital cutting," Culture, Health \& Sexuality 7(5): 463-477.

Department for International Development (DfID). 2012.

"How to Note. Guidance on monitoring and evaluation for programming on violence against women and Girls.

"https://assets.publishing.service.gov.uk/government/ uploads/system/uploads/attachment data/file/67334/How -to-note-VAWG-3-monitoring-eval.pdf.

World Health Organization (WHO). "Monitoring and evaluation basics." Geneva: WHO.

https://www.who.int/hiv/topics/vct/sw toolkit/monitoring and evaluation/en/

\section{References}

1. United Nations Children's Fund (UNICEF). 2016. Female Genital Mutilation/Cutting: A global Concern. New York: UNICEF; Available at: https://data.unicef.org/wpcontent/uploads/2016/04/FGMC-2016brochure 250.pdf

2. Askew, I. 2005. "Methodological issues in measuring the impact of interventions against female genital cutting," Culture, Health \& Sexuality 7(5): 463-477.

3. Department for International Development (DfID). 2012. "How to Note. Guidance on Monitoring and Evaluation for Programming on Violence against Women and Girls". Available at:

https://assets.publishing.service.gov.uk/government/u ploads/system/uploads/attachment data/file/67334/H ow-to-note-VAWG-3-monitoring-eval.pdf

4. World Health Organization (WHO). 2017. "Programme reporting standards for sexual, reproductive, maternal, newborn, child and adolescent health. "Geneva: WHO.

5. Workgroup for Intervention Development and Evaluation Research (WIDER) Group. "WIDER Recommendations to Improve Reporting of the Content of Behaviour Change Interventions." [accessed 18 December 2019]. Available from: https://bit.ly/2M8h1XJ

6. Pan American Health Organization (PAHO). Guidelines: Guide to Document Health Promotion Initiatives [Internet]. PAHO: Washington, DC; accessed 19 December 2019]. Available at: https://ctb.ku.edu/sites/default/files/chapter files/ quidelines guide to document health promotion initiatives.pdf

\section{Acknowledgements}

This publication was prepared by Caroline Kabiru of the Population Council. Thanks are due to Baker Maggwa, Francis Obare, Harriet Birungi, lan Askew, Kathryn Yount, and Michelle Hindin for their useful comments on an earlier draft of this guidance note.

This guidance note was developed with support from UK Aid from the UK government under the DfID research project "Evidence to End FGM/C: Research to Help Girls and Women Thrive," which is coordinated by the Population Council. All content is the sole responsibility of the authors and does not represent the opinions of the UK Government or the Population Council.

\section{Suggested citation:}

Evidence to End FGM/C: Research to Help Girls and Women Thrive Research Programme. 2020. "Guidance Note. Improving the rigor, relevance and utility of descriptions of FGM/C interventions and their evaluation." New York: Population Council. 